\title{
Dopamine-Imprinted Polymers: Template-Monomer Interactions, Analysis of Template Removal and Application to Solid Phase Extraction
}

\author{
Piotr Luliński ${ }^{1}$, Dorota Maciejewska ${ }^{1, *}$, Magdalena Bamburowicz-Klimkowska ${ }^{2}$ and Mirosław \\ Szutowski $^{2}$ \\ ${ }^{1}$ Department of Organic Chemistry, Faculty of Pharmacy, Medical University of Warsaw, Banacha 1 \\ Str., 02-097 Warsaw, Poland \\ 2 Department of Toxicology, Faculty of Pharmacy, Medical University of Warsaw, Banacha 1 Str., 02- \\ 097 Warsaw, Poland
}

* Author to whom correspondence should be addressed; E-mail: domac@farm.amwaw.edu.pl;

Tel: (+48) 225720643; Fax: (+48) 225720643.

Received: 21 August 2007; in revised form: 9 October 2007 / Accepted: 21 October 2007 / Published: 1 November 2007

\begin{abstract}
A dopamine-imprinted polymer (MIP) was prepared in aqueous methanol solution at $60^{\circ} \mathrm{C}$ by free-radical cross-linking polymerization of methacrylic acid in the presence of ethylene glycol dimethacrylate as the cross-linker and dopamine hydrochloride as the template molecule. Its ability to isolate dopamine was evaluated as the basis of a solid phase extraction procedure and compared with that of a non-imprinted polymer (NIP). The binding of dopamine was $84.1 \%$ and $29.1 \%$ for MIP and NIP, respectively. Various reported post-polymerization treatments to reduce template bleeding were examined. In our case the lowest bleeding was achieved after applying a combined procedure: continuous extraction in a Soxhlet apparatus (CE), followed by microwaveassisted extraction (ME) to a level of $0.061 \mu \mathrm{g} / \mathrm{mL}$. A simplified model of the templatemonomer complexes allowed rationalization of monomer choice based on the heats of complex formation at a PM3 level of theory.
\end{abstract}

Keywords: Dopamine hydrochloride isolation; theoretical binding energy; molecularly imprinted polymer; template removal; solid phase extraction 


\section{Introduction}

2-(3,4-Dihydroxyphenyl)ethylamine (dopamine, DA) is one of the most important neurotransmitters, involved in various physiological and pathophysiological processes modulated by its levels in various cerebral tissues [1-3]. In vivo studies aiming to define dopamine content in the brain have been hampered by its very low concentration (as low as $0.018-0.020 \mu \mathrm{g} / \mathrm{mL}$ ). Several research groups have investigated the problems of detection sensitivity and selective determination methods for dopamine. Those methods include fluorimetry [4, 5], spectrophotometry [6] and highly selective and sensitive determination with modified glassy carbon electrodes [7]. Recently, the molecular imprinting technique has been described as a versatile tool for cost-effective preparation of polymeric materials with affinity and selectivity towards specific desired molecules [8-10]. Molecularly imprinted polymers (MIPs) find applications in many areas such as bio-mimetic sensors [11], antibody mimics [12] and catalysis [13]. They are very useful materials for isolation and preconcentration of low concentration analytes employing the solid phase extraction (SPE) technique [14-16]. Although many papers have been published concerning utilization of MIPs in pharmaceutical science [17-19], only a few were devoted to dopamine isolation [20-24]. Recently, two scientific groups have published their results of the evaluation of dopamine imprinted polymers prepared using synthetic methods based on covalent [22] and non-covalent [23] approaches. It should be noted that the already-described dopamine imprinted polymers do not present high affinity and their properties were strongly dependent on temperature. For these reasons the search for new MIPs for dopamine isolation is still an interesting scientific goal. We decided to study the properties of a bulk polymer obtained on the basis of noncovalent interaction of functional monomer with DA hydrochloride (DAh). DAh is soluble in water, but not in the organic solvents used in standard applications of MIPs. Therefore, we used some water in the polymerization and solid phase extraction steps, hoping that this would help us to overcome the problem. We also concentrated on the problem of removal of the template molecule from the imprinted polymer, before using it as a resin in the SPE technique.

Recently, several scientific groups have developed computational approaches to preselect the functional monomers used in the synthesis of MIPs [25-28]. In this paper we report a simple and facile theoretical analysis of the intermolecular interactions in the complexes between dopamine hydrochloride as a template and two functional monomers - acrylonitrile (AN) and methacrylic acid (MAA) - assuming that a higher energy of binding, $\Delta \mathrm{E}$, could indicate a more suitable monomer for use in the synthetic procedure. On this basis we chose the functional monomer and prepared the resin for SPE. We report various post-polymerization treatments of the imprinted polymer aimed at elimination of DAh bleeding at a level that would allow a direct use of MIPs for analytical purposes. We also describe a new and efficient SPE procedure.

\section{Results and Discussion}

\section{Molecular modeling of complexes}

The structures of all the considered complexes of dopamine cation with methacrylic acid (MAA) and acrylonitrile (AN), corresponding to local minima are shown in Figure 1, and relevant binding energies $\Delta \mathrm{E}$ are given in Table 1 . In both complexes analyzed without water, named MA1 and A1, 
monomer heteroatoms and DA are involved in hydrogen bonds with a length of $1.8 \AA$. Only one hydrogen bond in the $\mathrm{A} 1$ complex is longer by $0.6 \AA$. Both these complexes exhibit high $\Delta \mathrm{E}$ values (MA1: -175.49 kJ/mol; A1: -155.29 kJ/mol) and their formation is favorable.

Figure 1. The complexes formed between dopamine cation - methacrylic acid, (MA1 without water and MA2 in water), and dopamine cation - acrylonitrile (A1 without water and A2 with water). Dotted lines indicate the hydrogen bonds.
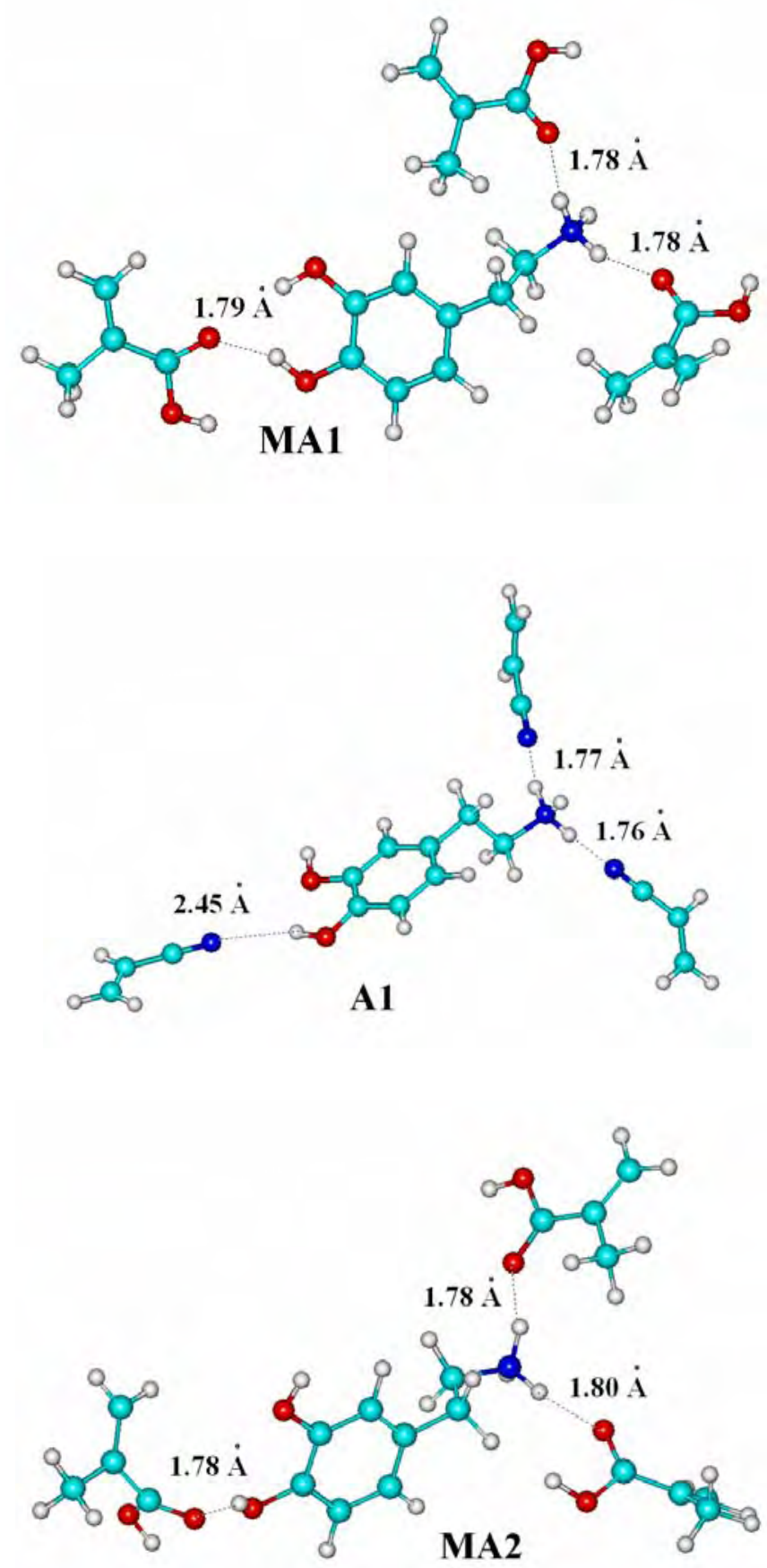
Fig.1 cont.
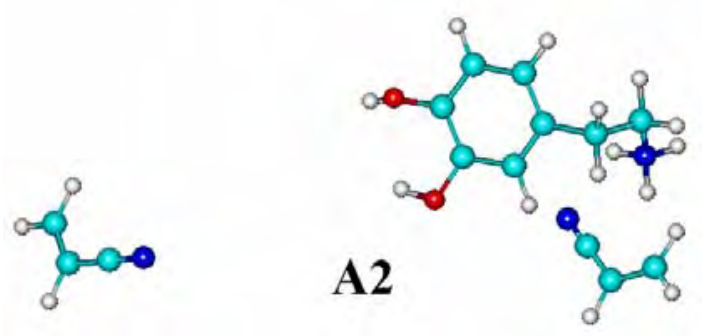

Table 1. Binding energies $\Delta \mathrm{E}$ of dopamine cation with methacrylic acid (MA) and acrylonitrile (A).

\begin{tabular}{cc}
\hline Complexes & Energy $\Delta \mathbf{E}\left[\mathbf{k J} \cdot \mathbf{m o l}^{\mathbf{1}}\right]$ \\
\hline MA1 & -175.49 \\
MA2 (in water) & -151.18 \\
A1 & -155.29 \\
A2 (in water) & -49.51 \\
\hline
\end{tabular}

In the complexes formed in water, named MA2 and A2, the $\mathrm{H}_{2} \mathrm{O}$ molecules disrupt the existing hydrogen bonds and destabilize both complexes in a different way. The DA cation complex with the methacrylic acid (MA2) is still stable $(-151.83 \mathrm{~kJ} / \mathrm{mol})$, but the stability of the acrylonitrile complex (A2) has decreased because its energy increased to $-49.51 \mathrm{~kJ} / \mathrm{mol}$, indicating that water molecules participate in hydrogen bonds instead of acrylonitrile (see the long distances between AN and the DA cation in complex A2 in Figure 1). Consequently, the synthetic procedure, carried out in the presence of water molecules, is expected to give a more selective resin with MAA as a functional monomer used. The binding affinities of imprinted polymers obtained with methacrylic acid or with acrylonitrile as monomers (see below) support these findings.

\section{Preparation of molecularly imprinted polymers}

Two kinds of MIPs and the corresponding control NIPs were prepared using two monomers, methacrylic acid (MAA) or acrylonitrile (AN). The thermal method involving free-radical polymerization [29] with dopamine hydrochloride as a template was chosen.

The crucial problem for polymerization results was the selection of the appropriate solvent, named porogen. It is known that the porogen determines the strength of non-covalent interaction and affects polymer morphology. DAh is insoluble in non-polar, aprotic solvents used as porogens, but it is soluble in water and in mixtures of water and acetonitrile or water and methanol. Our previous analysis [30] of binding properties of MIP based on MAA showed that the use of an aprotic and weakly polar solvent such as chloroform was inappropriate. The results for a mixture of water and acetonitrile (15:85 v/v) as porogen (see binding values in Table 2) were also non-promising, because of highly non-specific binding. Only the application of a mixture of water and methanol afforded a resin with 
specific dopamine binding sites. The change in the adsorption level of dopamine with a decrease in the $\mathrm{pH}$ value of the conditioning solution could be related to ionic equilibria between dopamine cation and carboxylate groups in the polymer matrix. We could suppose that at $\mathrm{pH} 5$ the intermolecular interactions between DAh and C=O fragment of MIP are more specific. We carefully set the amount of water in the porogen mixture at $12.5 \%$. It is the lowest amount of water necessary to dissolve the DAh. After the grinding, sieving and suspending processes, the obtained materials were dried in oven at $50^{\circ} \mathrm{C}$ overnight and used in subsequent treatments.

Table 2. Binding of dopamine hydrochloride using imprinted (MIP) and non-imprinted (NIP) polymers prepared in different porogen systems with different resin conditioning.

\begin{tabular}{|c|c|c|c|c|c|}
\hline \multicolumn{6}{|c|}{ Binding (\%) $\left.{ }^{a}\right)$} \\
\hline \multicolumn{2}{|c|}{ Porogen system } & \multicolumn{2}{|c|}{ water-acetonitrile $(15: 85, \mathrm{v} / \mathrm{v})$} & \multicolumn{2}{|c|}{ water-methanol $(12.5: 87.5, \mathrm{v} / \mathrm{v})$} \\
\hline Condi & & pH 5 & $\mathrm{pH} 8$ & pH 5 & $\mathrm{pH} 8$ \\
\hline \multirow{2}{*}{ Load } & MIP & 71.4 & 100.0 & 84.1 & 98.3 \\
\hline & NIP & 59.2 & 100.0 & 29.1 & 91.7 \\
\hline
\end{tabular}

Preliminary binding studies of polymers confirmed our theoretical analysis. Affinity towards DAh of acrylonitrile polymers were $18.7 \%$ for MIP and $13.9 \%$ for NIP. Only the polymers based on MAA were used for the subsequent experiments.

\section{Template removal}

Once the imprinted polymer was obtained, it had to be cleaned in order to remove the template molecule leaving three-dimensional cavities ready for analyte binding. The residual deposition of the template is a well-known problem of molecularly imprinted polymers, which could affect the accuracy of analytical results [31]. We employed four different techniques (i-iv) to remove the template from polymer matrix: i) continuous extraction using a Soxhlet apparatus (CE), ii) microwave-assisted extraction (ME), iii) stationary extraction (SE), and iv) permanent elution (PE).

After treatments $i$ and $i$ the elution solvent, $0.05 \mathrm{M}$ aqueous ammonium formate adjusted to $\mathrm{pH} 3$ - methanol $(3: 1 \mathrm{v} / \mathrm{v})$ was chosen to determine the level of dopamine bleeding. Our results show that the use of CE procedure resulted in bleed levels of $0.405 \mu \mathrm{g} / \mathrm{mL}$. ME more than halved that amount to $0.176 \mu \mathrm{g} / \mathrm{mL}$. We also combined both methods, i.e., CE followed by ME, which lowered the template concentration to $0.061 \mu \mathrm{g} / \mathrm{mL}$. The dopamine bleeding could be reduced to $0.031 \mu \mathrm{g} / \mathrm{mL}$ by extending of CE procedure with acetonitrile, but the cost of this additional stage is high. The binding parameter of NIP particles exposed for microwave irritation showed no significant differences when compared with the corresponding untreated NIP, indicating that the polymer morphology did not change during microwave treatment.

The effects of different time of desorption on the amount of dopamine removed from polymer matrix were tested according to SE procedure. The results are shown in Figure 2. As can be seen, the maximum of DA release was at $24 \mathrm{~h}$, but this value is only slighly higher to the amount desorbed after 
$6 \mathrm{~h}, 122.1$ vs. $118.3 \mu \mathrm{g} / \mathrm{mL}$, respectively. Therefore, the operation time over $6 \mathrm{~h}$ has no significant effect on desorption of dopamine.

Figure 2. Concentration of dopamine released from polymer in stationary extraction experiments (SE), $(n=3)$.

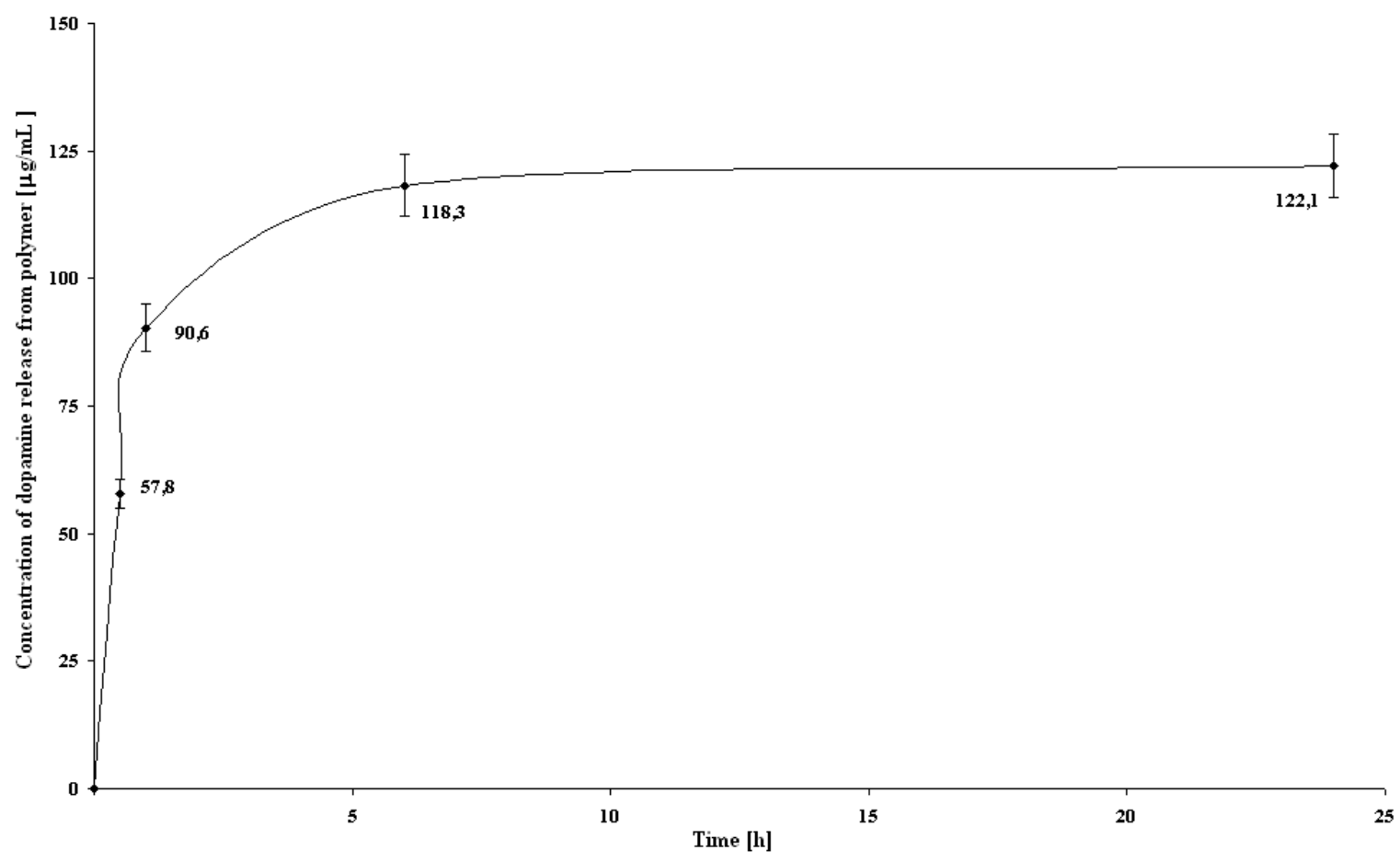

In order to examine the amount of template removed from polymer matrix during permanent elution, we applied PE procedure at various conditions shown in Table 3.

Table 3. Experimental details of permanent elution (PE).

\begin{tabular}{ccccc}
\hline $\begin{array}{c}\text { Number of } \\
\text { protocol/column }\end{array}$ & $\begin{array}{c}\text { Volume } \\
\text { of eluant } \\
{[\mathbf{m L}]^{\mathbf{a})}}\end{array}$ & $\begin{array}{c}\text { Interval between } \\
\text { elution steps } \\
\text { [min] }\end{array}$ & $\begin{array}{c}\text { Total time of } \\
\text { ten elution steps } \\
\text { [min] }\end{array}$ & Drying \\
\hline S 1 & 1 & 5 & 60 & $\begin{array}{c}\text { No special } \\
\text { treatment }\end{array}$ \\
\hline S 2 & 25 & 5 & 300 & $\begin{array}{c}\text { No special } \\
\text { treatment }\end{array}$ \\
\hline S 3 & 1 & 30 & 310 & $\begin{array}{c}\text { No special } \\
\text { treatment }\end{array}$ \\
\hline S 4 & 1 & 5 & 60 & $\begin{array}{c}\text { Nitrogen } \\
\text { stream }\end{array}$ \\
\hline S 5 & 1 & 5 & 60 & $\begin{array}{c}\text { Hot air } \\
\text { stream }\end{array}$ \\
\hline
\end{tabular}

a) Flow rate: $1 \mathrm{~mL} / \mathrm{min}$ 
Three potentially important parameters were tested: the amount of eluent, the interval time between elution steps and the drying method (flow speed was held constant at $1 \mathrm{~mL} / \mathrm{min}$ ). Five kinds of samples represent various protocols designated as S1-S5. The relationship between the concentration of DA removed from MIP $(\mu \mathrm{g} / \mathrm{mL})$ and the number of subsequent elution steps is shown in Figure 3.

Figure 3. Concentration of dopamine eluted from polymer in S1 - S5 permanent elution protocols (PE). R.S.D. were lower than $4 \%$ in all instances $(n=3)$.

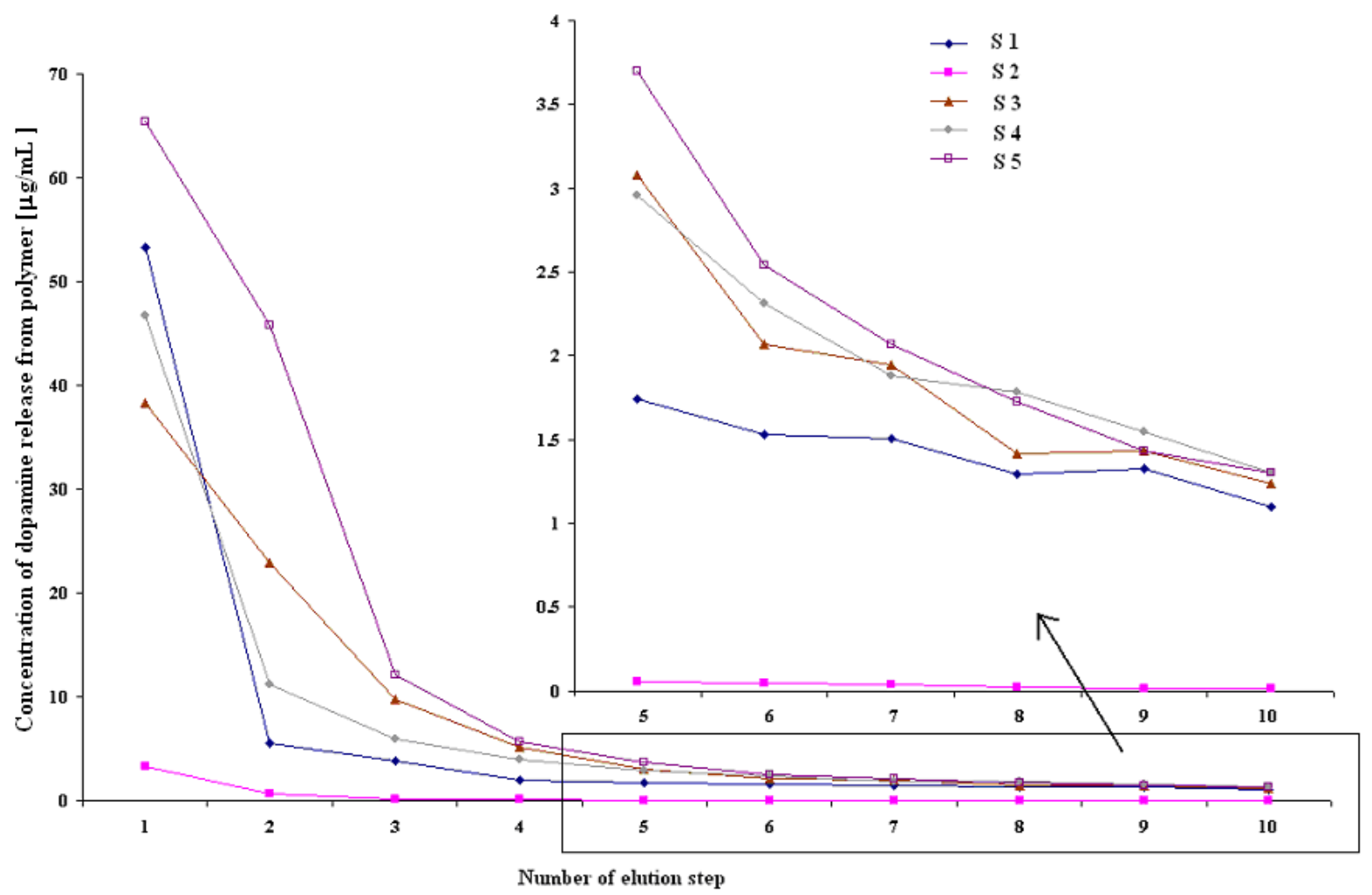

All the tested parameters play an important role in template removal. After each step a smaller amount of the template was removed from MIP. For all the protocols, except S2, the concentrations obtained after 10 steps were in the range between 1.1 - $1.3 \mu \mathrm{g} / \mathrm{mL}$. Protocol S2, when $25 \mathrm{~mL}$ of solvent was used, gave very low concentrations after 10 steps, but the total amount of DA eluted from the resin was similar to that seen in other protocols (S1: $73.2 \mu \mathrm{g}, \mathrm{S} 2: 104.7 \mu \mathrm{g}, \mathrm{S} 3: 87.3 \mu \mathrm{g}, \mathrm{S} 4: 79.5 \mu \mathrm{g}, \mathrm{S} 5$ : $141.7 \mu \mathrm{g})$, implying that the use of larger volumes of eluent is impractical for template removal from the resin. It can also be seen that drying intervals between consecutive elutions give the highest total amount of dopamine eluted from the resin (protocol S5). Protocol S1 repeated after 3 days showed a significant increase of template removal from polymer matrix (Figure 4). Although many individual elution protocols were used, PE procedure was found to be ineffective in quantitative removal of the template molecule and impractical because the use of large volumes of eluent that were required. 
Figure 4. Concentration of dopamine eluted in long-term extraction. R.S.D. were lower than $4 \%$ in all instances $(n=3)$.

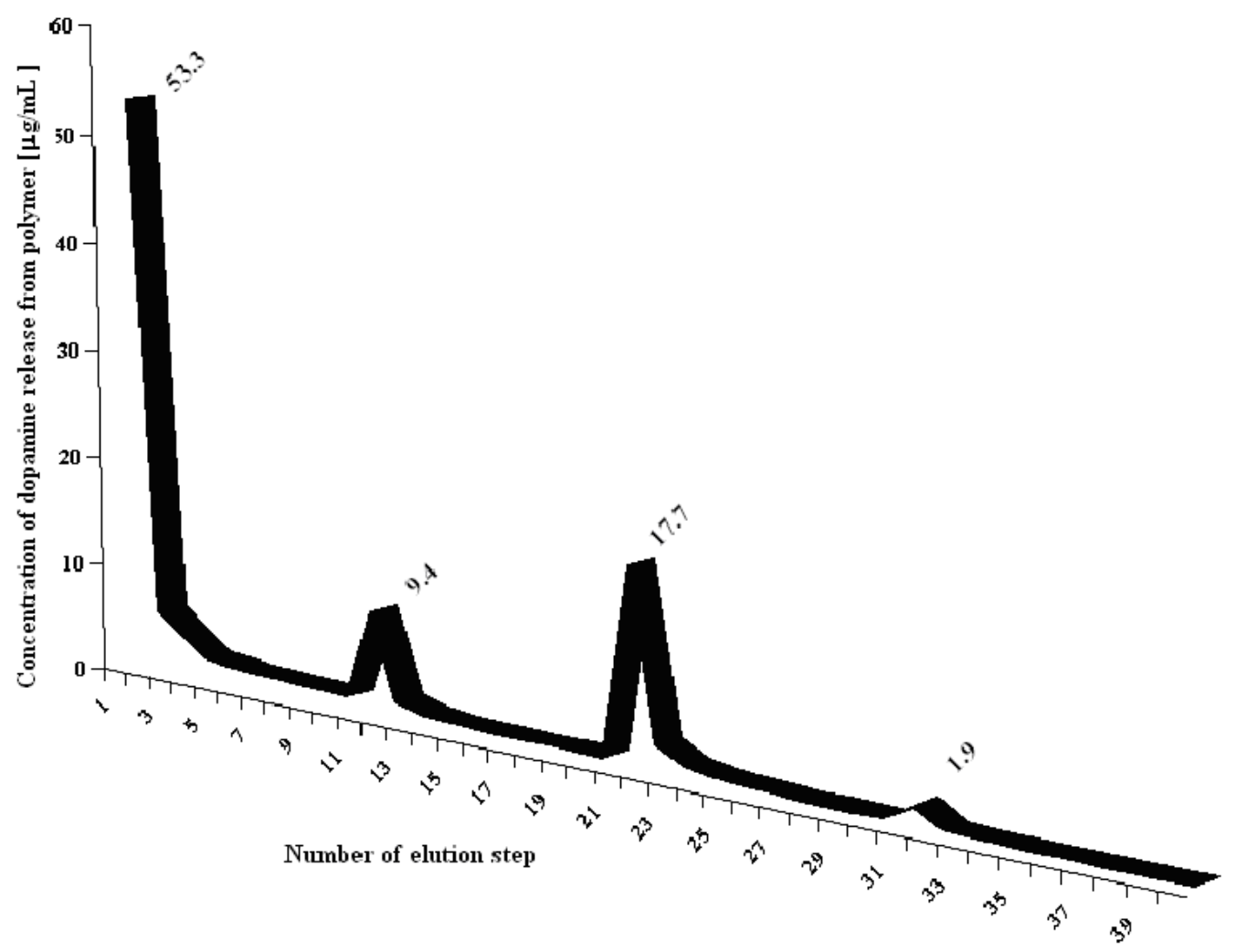

In conclusion, the application of the combined CE/ME procedures resulted in the most efficient template removal.

\section{Solid-phase extraction}

We have examined the influence of $\mathrm{pH}$ of the conditioning buffer for further binding of DAh on the resins polymerized with the water - methanol mixture as a porogen. Binding (expressed as a ratio of the amount of DAh adsorbed by resin to the total amount of DAh in standard solution, applied on resin) of DAh adsorption after conditioning step at $\mathrm{pH}$ 8, on both MIP and NIP, was high at 98.3\% for MIP and $91.7 \%$ for NIP. There was also no significant difference between MIP and NIP during further steps of SPE. Binding of DAh after conditioning step at $\mathrm{pH} 5$ resulted in bigger differences in adsorption between MIP and NIP and was 84.1\% for MIP and 29.1\% for NIP. The efficiency, expressed as the recovery (a ratio of the amount of DAh in the eluent to the total amount of DAh in standard solution, applied on resin loaded) of DAh in subsequent extraction stages are given in Table 4. Total amount of dopamine desorbed from polymer exceed one hundred percent and may suggest some template leakage. 
Table 4. Efficiency of load, wash and elute steps of dopamine hydrochloride (concentration: $1 \mu \mathrm{g} / \mathrm{mL}$, in water, volume: $1 \mathrm{~mL}$ ).

\begin{tabular}{lcc}
\hline \multirow{2}{*}{ Step } & \multicolumn{2}{c}{ Recovery $( \pm$ S.D., $\boldsymbol{n}=\mathbf{5 ,}$ \%) } \\
\cline { 2 - 3 } & MIP & NIP \\
\hline Loading & $15.9 \pm 3.7$ & $70.8 \pm 2.4$ \\
Washing & $17.3 \pm 1.6$ & $10.6 \pm 2.2$ \\
Elution 1 & $62.3 \pm 3.1$ & $10.6 \pm 2.2$ \\
Elution 2 & $6.9 \pm 1.3$ & $3.3 \pm 0.9$ \\
\hline
\end{tabular}

Finally, we examined imprinted resin for competitive extraction of mixture of three catecholoamines: dopamine, epinephrine, norepinephrine and the related neurotransmitter 5-hydroxytryptamine. The structures of the tested molecules are shown in Figure 5.

Figure 5. Chemical structures of dopamine (I), epinephrine (II), norepinephrine (III) and 5-hydroxytryptamine (IV).<smiles>NCCc1ccc(O)c(O)c1</smiles>

(I)<smiles>NCC(O)c1ccc(O)c(O)c1</smiles>

(III)<smiles>CNCC(O)c1ccc(O)c(O)c1</smiles>

(II)<smiles>NCCc1c[nH]c2ccc(O)cc12</smiles>

(IV)

Surprisingly, our results showed the highest competitive adsorption for 5-hydroxytryptamine (32.5\%). Three catecholoamines were also adsorbed: epinephrine (23.1\%), dopamine (20.9\%) and norepinephrine (13.5\%). The higher adsorption of 5-hydroxytryptamine as compared to the catecholamines, might be explained by the presence of an additional $\mathrm{N}$ atom in the analyte molecule, which could be more efficient in its interaction with the polymer matrix. Results of subsequent extraction steps are given in Table 5. 
Table 5. Efficiency of load, wash and elute steps of epinephrine, norepinephrine and 5-hydroxytryptamine (concentration: $1 \mu \mathrm{g} / \mathrm{mL}$, mixture of each in water, volume: $1 \mathrm{~mL}$ ).

\begin{tabular}{lccc}
\hline \multirow{2}{*}{ Step } & \multicolumn{3}{c}{ Recovery ( \pm S.D., $\boldsymbol{n}=\mathbf{5 ,}$ \%) } \\
\cline { 2 - 4 } & Epinephrine & Norepinephrine & 5-Hydroxytryptamine \\
\hline Loading & $76.8 \pm 5.0$ & $86.5 \pm 4.3$ & $67.5 \pm 1.0$ \\
Washing & $5.2 \pm 2.4$ & $7.8 \pm 0.9$ & $12.0 \pm 0.4$ \\
Elution 1 & $2.9 \pm 1.4$ & $4.9 \pm 2.2$ & $9.3 \pm 5.1$ \\
Elution 2 & $0.2 \pm 0.1$ & $0.3 \pm 0.1$ & $3.7 \pm 0.6$ \\
\hline
\end{tabular}

\section{Conclusions}

We have demonstrated the utility of theoretical analyses of prepolymerization complexes for the rational development of an effective dopamine recognizing resin. This simple and facile approach could indicate an appropriate functional monomer. The analysis of several post-treatment procedures revealed that the combined $\mathrm{CE} / \mathrm{ME}$ approach with the use of $\mathrm{pH} 3$ aqueous ammonium formate methanol (3:1, v/v) gave the lowest bleed level for dopamine imprinted polymer $(0.061 \mu \mathrm{g} / \mathrm{mL})$. After post-polymerization treatments DAh was strongly bound by MIP (84.1\%), in contrast to NIP (29.1\%). A new SPE protocol allowed an efficient elution of dopamine hydrochloride from MIP. During a competitive extraction procedure the MIP adsorbed 5-hydroxytryptamine (32.5\%), epinephrine (23.1\%), dopamine (20.9\%) and norepinephrine (13.5\%).

\section{Experimental}

\section{General}

Dopamine hydrochloride, epinephrine bitartrate salt, norepinephrine bitartrate salt and 5-hydroxytryptamine hydrochloride was obtained from Sigma - Aldrich (Germany). Ammonium formate, formic acid, sodium dihydrogen orthophosphate and orthophosphoric acid, acetone and isopropanol were supplied by POCh Gliwice (Poland). Perchloric acid was from Veb Laborchemie Apolda (Germany). Methacrylic acid, acrylonitrile, ethylene glycol dimethacrylate, 2,2'-azobis(isobutyronitrile) were purchased from Fluka (Germany) and 2,2'-azobis[2-(2-imidazolin-2-yl)propane] dihydrochloride was purchased from Wako Pure Chemical Industries, Ltd. HPLC - grade acetonitrile and methanol were from Labscan (Ireland). The monomers were purified prior to use by standard procedures in order to remove stabilizers. 2,2'-azobis(isobutyronitrile) was recrystallized from ethanol. All other reagents were used without further purification. Ultra-pure water was obtained from a Milli-Q purification system (Millipore, France). Microwave-supported extraction of the template was performed in a Plazmatronika Microwave Oven, 900W (Plazmatronika, Poland). Solid phase extraction was performed using a Bakerbond 12-column vacuum manifold and $1 \mathrm{~mL}$ SPE glass columns (J.T. Baker, The Netherlands). The HPLC system used consisted of a Model LC - 10 AT pump, a Model CTO 10A oven, a Model RF 551 spectrofluorimetric detector operated at $\lambda_{\mathrm{EX}}=280 \mathrm{~nm}$ and $\lambda_{\mathrm{EM}}=315 \mathrm{~nm}$ [32] and $20 \mu \mathrm{L}$ injector - Rheodyne 7725 (Shimadzu, Germany). Chromatographic separation was performed using a Discovery HS F5 stainless steel column (150 mm x 4.6 mm ID, $5 \mu \mathrm{m}$, Supelco, 
Bellefonte, PA, USA), preceded by a $20 \mathrm{~mm}$ x $4.6 \mathrm{~mm}$ ID, Discovery HS F5 guard column. A reversed phase HPLC method was used for the quantitative analysis of dopamine. The column was heated at $40^{\circ} \mathrm{C}$. The mobile phase consisted of $0.05 \mathrm{M} \mathrm{pH} 3$ aqueous ammonium formate - methanol solution $(84: 16 \mathrm{v} / \mathrm{v})$, delivered at a flow rate of $1.0 \mathrm{~mL} / \mathrm{min}$ [33]. The mobile phase was ultrasonically degassed prior to use. The six point calibration curve was linear ranging between $0.05 \mu \mathrm{g} / \mathrm{mL}$ and 1 $\mu \mathrm{g} / \mathrm{mL}$ with correlation coefficients $\mathrm{r}^{2}=0.999$ and the limit of detection (LOD) was $0.044 \mu \mathrm{g} / \mathrm{mL}$. Fluorescence measurements of DA hydrochloride content were performed with a RF-5001PC spectrophotofluorometer equipped with a $150 \mathrm{~W}$ xenon lamp (Shimadzu, Germany). Intensity was measured at $315 \mathrm{~nm}$ using an excitation wavelength of $280 \mathrm{~nm}$. The calibration curve (fluorescence (y) versus concentration ( $\mathrm{x}$ ) was constracted in the range of $0.1 \mu \mathrm{g} / \mathrm{mL}$ to $3 \mu \mathrm{g} / \mathrm{mL}$. The calibration equation was: $y=27.12 x+1.48, r^{2}=0.996$, and the limit of detection (LOD) was $0.047 \mu \mathrm{g} / \mathrm{mL}$.

\section{Stock solutions of catecholamines and 5-hydroxytryptamine}

In a $100 \mathrm{~mL}$ volumetric flask an accurately weighed amount of dopamine hydrochloride $(0.0062$ g), norepinephrine bitartrate salt $(0.0100 \mathrm{~g})$, epinephrine bitartrate salt (0.0091 g) or 5-hydroxytryptamine hydrochloride $(0.0060 \mathrm{~g})$ standard was dissolved in ultra-pure water adjusted to $\mathrm{pH} 3$ with $0.04 \mathrm{M}$ perchloric acid. The obtained concentration was $50 \mu \mathrm{g} / \mathrm{mL}$. The stock solutions of catecholamines and 5-hydroxytryptamine hydrochloride were stored at $4^{\circ} \mathrm{C}$. The stock standards solution was mixed in one solution or diluted separately to a finale concentration of $1 \mu \mathrm{g} / \mathrm{mL}$ before being used.

Procedure for the preparation of molecularly imprinted polymer (MIP) with methacrylic acid as functional monomer: Dopamine hydrochloride (0.063 g, $0.33 \mathrm{mmol})$, methacrylic acid (0.227 g, 2.64 mmol), ethylene glycol dimethacrylate (3.96 g, $20 \mathrm{mmol}$ ) and 2,2'-azobis(isobutyronitrile) (65 mg) were mixed with a solution of methanol $(3.67 \mathrm{~mL})$ and water $(0.53 \mathrm{~mL})$. The mixture was purged with nitrogen for $5 \mathrm{~min}$, and polymerized by heating at $60^{\circ} \mathrm{C}$ for $24 \mathrm{~h}$. The resulting monolith was ground in a mortar with a pestle and wet-sieved into particles below $45 \mu \mathrm{m}$ diameter. Fine particles were removed by repeated decantation in acetone. Finally, the particles were extracted to remove the template (for details see below), dried in oven at atmospheric pressure at $50^{\circ} \mathrm{C}$ and used for further examinations. The control, non-imprinted polymer (NIP) was synthesized and treated in the same way as the corresponding imprinted polymer, except that no DA hydrochloride was added.

Procedure with acrylonitrile as functional monomer: Dopamine hydrochloride (0.063 g, $0.33 \mathrm{mmol})$, acrylonitrile (0.140 g, $2.64 \mathrm{mmol})$, ethylene glycol dimethacrylate (3.96 g, $20 \mathrm{mmol}$ ) and 2,2'azobis[2-(2-imidazolin-2-yl)propane] dihydrochloride (55 mg) were mixed with a solution of methanol (3.67 $\mathrm{mL})$ and water $(0.53 \mathrm{~mL})$. The mixture was purged with nitrogen for $5 \mathrm{~min}$ and polymerized by heating at $45^{\circ} \mathrm{C}$ for $24 \mathrm{~h}$. The resulting monolith was ground in a mortar with a pestle and wet-sieved into particles below $45 \mu \mathrm{m}$ diameter. Fine particles were removed by repeated decantation in acetone. Finally, the particles were extracted to remove the template with combined CE/ME procedures (see below), dried in oven at atmospheric pressure at $50^{\circ} \mathrm{C}$ and used for further examinations. The control, non-imprinted polymer (NIP) was synthesized and treated in the same way as the corresponding imprinted polymer, except that no DA hydrochloride was added. 


\section{Template removal}

Four different strategies were used to remove the template molecule from polymeric network: $i$ ) continuous extraction using a Soxhlet apparatus (CE), ii) microwave-assisted extraction (ME), iii) stationary extraction (SE), iv) permanent elution (PE).

i) Continuous extraction using a Soxhlet apparatus (CE): MIP particles (439 mg) were placed in a Soxhlet apparatus and continuously extracted (30 cycles, $80 \mathrm{~mL}$, methanol - water, 1:1 v/v). Before further analysis, particles were dried at $50^{\circ} \mathrm{C}$ in an oven at atmospheric pressure.

ii) Microwave-assisted extraction (ME): MIP particles (415 mg) were put into a microwave vessel, and methanol - water $(1: 1 \mathrm{v} / \mathrm{v}, 10 \mathrm{~mL})$ was added; the mixture was refluxed with internal stirring for $2 \mathrm{~min}$, then cooled for $2 \mathrm{~min}$. This step was repeated twice, then the particles were filtered off and transferred to the vessel. The procedure was repeated twice with a fresh portion of the solvent mixture, then the solvent system was changed to isopropanol and once more to methanol - water, 1:1 v/v, and the same procedure was followed. Finally, the vessel was allowed to reach room temperature and its contents were collected and dried at $50^{\circ} \mathrm{C}$ in an oven at atmospheric pressure. To compare the influence of microwave radiation on polymer network structure, a sample of non-imprinted polymer (NIP) was also treated once with each solvent system according to the given procedure. To determine the level of dopamine bleeding from resin after treatments $i$ and $i$, we proceeded as follows: dry MIP samples (56.2 mg) were put into $1 \mathrm{~mL}$ glass SPE columns and eluted with $\mathrm{pH} 3$ ammonium formate, methanol solution ( $3: 1 \mathrm{v} / \mathrm{v}, 1 \mathrm{~mL})$. An aliquot of solvent $(400 \mu \mathrm{L})$ was used to analyze the amount of bleeding DA by fluorimetry.

iii) Stationary extraction (SE): MIP particles (56.2 mg) were placed in Eppendorf vials and pH 3 ammonium formate - methanol solution $(3: 1 \mathrm{v} / \mathrm{v}, 1 \mathrm{~mL})$ was added to each sample. Vials were put into a shaker, and shaken out over various periods of time ranging from half an hour to $24 \mathrm{~h}$. Then the samples were centrifuged ( $2500 \mathrm{rpm}, 5 \mathrm{~min})$. An aliquot of solvent ( $400 \mu \mathrm{L}$ ) was used to analyze the amount of desorbed DA by fluorimetry.

iv) Permanent elution (PE): Five kinds of $1 \mathrm{~mL}$ glass SPE columns with dry packed MIP (56.2 mg) were eluted with $\mathrm{pH} 3$ ammonium formate - methanol solution $(3: 1 \mathrm{v} / \mathrm{v}$, flow rate $1 \mathrm{~mL} / \mathrm{min})$ in different ways S1 - S5, as shown in Table 3. An aliquot of solvent (400 $\mu \mathrm{L})$ was used to detect template concentration by the fluorescence spectroscopic method. Each experiment was performed in triplicate. To study the template removal for a long period of time, PE protocol of S1 (see Table 1) was repeated three times: after 3 h, 72 h and 75 h (between the experiments sample S1 was closed and stored in a refrigerator).

\section{Binding energy calculations}

Binding energy $(\Delta \mathrm{E})$ calculations were performed on a PM3 semiempirical level of theory with the chemically useful accuracy for molecules with $\mathrm{C}, \mathrm{H}, \mathrm{N}, \mathrm{O}$ atom bonds using the HyperChem 7.02 program [34]. These numerical results can be considered useful for qualitative comparisons among a 
series of similar complexes. It is obvious that in solution the pre-polymerization complexes could exist with a varying number of monomer molecules. We decided to analyze the complexes with the molecular ratio of DA cation to monomer equal to 1:3, as a minimum required for a three-point handle, suitable for cavities with good recognition places in the polymer.

The starting geometries of the complexes with both monomers, MAA and AN, were generated by placing four optimized molecules, DA cation and three MAA or three AN in the same file. The position of each molecule was chosen in such a way that the starting distances between the atoms that could potentially form hydrogen bonds were equal to $2 \AA$. Then the optimization of those files was performed, and heats of formation were defined. The binding energies of the complexes were

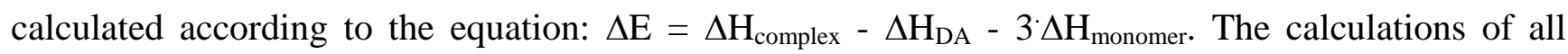
components were repeated in water boxes at the same level of theory using modules accessible in the HyperChem 7.02 program. Next, the energies of the optimized structures were computed using a single point procedure after removing the water molecules, and used in calculations of the binding energies of the complexes.

\section{Solid-phase extraction}

Glass columns (1 mL) were dry packed with MIP particles (56.2 mg), secured by PTFE frits, and used for the SPE. Another $1 \mathrm{~mL}$ glass column with NIP particles (56.2 mg) was prepared, and used for comparison.

Affinity study: A new protocol was worked out to select conditioning solutions. Additional steps of the solid phase extraction were the same as our work-up described earlier [30]. Procedures are listed below:

\section{Procedure 1}

1. Prewashing the particles with water $(1 \mathrm{~mL})$.

2. Conditioning with $\mathrm{pH} 8$ phosphate buffered saline $(2 \mathrm{~mL})$.

3. Loading with $1 \mu \mathrm{g} / \mathrm{mL}$ concentration of dopamine hydrochloride $(1 \mathrm{~mL})$.

4. Washing with water $(1 \mathrm{~mL})$.

5. Elution stages ( 1 and 2 ) were performed using $0.05 \mathrm{M}$ aqueous ammonium formate, $\mathrm{pH} 3$ methanol solution $(75: 25 \mathrm{v} / \mathrm{v}, 0.5 \mathrm{~mL})$.

\section{Procedure 2}

1. Prewashing the particles with water $(1 \mathrm{~mL})$.

2. Conditioning with water (2 mL) adjusted to $\mathrm{pH} 5$ with $0.04 \mathrm{M}$ perchloric acid.

3. Loading with $1 \mu \mathrm{g} / \mathrm{mL}$ concentration of dopamine hydrochloride $(1 \mathrm{~mL})$.

4. Washing with water $(1 \mathrm{~mL})$.

5. Elution stages ( 1 and 2 ) were performed using $0.05 \mathrm{M}$ aqueous ammonium formate, $\mathrm{pH} 3$ methanol solution $(75: 25 \mathrm{v} / \mathrm{v}, 0.5 \mathrm{~mL})$.

The eluates from each step were collected and $20 \mu \mathrm{L}$ used for the RP-HPLC analytical quantification. 
Selectivity study: Procedure 2 (for details see above) was adapted for selectivity study with loading stage as follows: column was loaded with catecholamine mixture $(1 \mu \mathrm{g} / \mathrm{mL}$ concentration, $1 \mathrm{~mL})$. The eluates from each step were collected and $20 \mu \mathrm{L}$ was used for the analytical RP-HPLC quantification.

\section{References}

1. Jaber, M.; Robinson, S. W.; Missale, C.; Caron, M. G. Dopamine receptors and brain function. Neuropharmacology 1996, 35, 1503-1519.

2. Wilson, J. M.; Sanyal, S.; Van Tol, H. H. M. Dopamine $\mathrm{D}_{2}$ and $\mathrm{D}_{4}$ receptor ligands: relation to antipsychotic action. Eur. J. Pharmacol. 1998, 351, 273-286.

3. Wong, A. H. C.; Buckle, C. E.; Van Tol, H. H. M. Polymorphisms in dopamine receptors: what do they tell us? Eur. J. Pharmacol. 2000, 410, 183-203.

4. Wang, H. Y.; Sun, Y.; Tang, B. Study on fluorescence property of dopamine and determination of dopamine by fluorimetry. Talanta 2002, 57, 899-907.

5. Wang, H. Y.; Hui, Q. S.; Xu, L. X.; Jiang, J. G.; Sun, Y. Fluorimetric determination of dopamine in pharmaceutical products and urine using ethylene diamine as the fluorigenic agent. Anal. Chim. Acta 2003, 497, 93-99.

6. Mamiński, M.; Olejniczak, M.; Chudy, M.; Dybko; Brzózka, Z. Spectrophotometric determination of dopamine in microliter scale using microfluidic system based on polymeric technology. Anal. Chim. Acta 2005, 540, 153-157.

7. Wang, H.-S.; Li, T.-H.; Jia, W.-L.; Xu, H.-Y. Highly selective and sensitive determination of dopamine using a Nafion/carbon nanotubes coated poly(3-methylthiophene) modified electrodes. Biosens. Bioelectron. 2006, 22, 664-669.

8. Komiyama, M.; Takeuchi, T.; Mukawa, T.; Asanuma, H. (Eds.) Molecular Imprinting: From Fundamentals to Applications; Wiley-VCH: Weinheim, 2003.

9. Haupt, K. Molecularly imprinted polymers and their use in biomimetics sensors. Chem. Rev. 2000, 100, 2495-2504.

10. Wuff, G. Enzyme-like catalysis by molecularly imprinted polymers. Chem. Rev. 2002, 102, 1-28.

11. Rick, J.; Chou, T. C. Amperometric protein sensor - fabricated as a polypyrrole, polyaminophenylboronic acid bilayer. Biosens. Bioelectron. 2006, 22, 329-335.

12. Haupt, K. Imprinted polymers - tailor-made mimics of antibodies and receptors. Chem. Commun. 2003, 171-178.

13. Alexander, C.; Davidson, L.; Hayes, W. Imprinted polymers: artificial molecular recognition materials with applications in synthesis and catalysis. Tetrahedron 2003, 59, 2025-2057.

14. He, C. Y.; Long, Y. Y.; Pan, J. L.; Li, K.; Lui, F. Application of molecularly imprinted polymers to solid-phase extraction of analytes from real samples. J. Biochem. Biophys. Meth. 2007, 70, 133-150.

15. Qiao, F. X.; Sun, H. W.; Yan, H. Y.; Row, K. H. Molecularly imprinted polymers for solid phase extraction. Chromatographia 2006, 64, 625-634.

16. Yang, J.; Hu, Y.; Cai, J.-B.; Zhu, X.-L.; Su, Q.-D. A new molecularly imprinted polymer for selective extraction of cotinine from urine samples by solid phase extraction. Anal. Bioanal. Chem. 2006, 384, 761-768. 
17. Svenson, J.; Nicholls, I. A. On the thermal and chemical stability of molecularly imprinted polymers. Anal. Chim. Acta 2001, 435, 19-24.

18. Owens, P. K.; Karlsson, L. Molecular imprinting for bio- and pharmaceutical analysis. Trends Anal. Chem. 1999, 18, 146-154.

19. Andersson, L. I. Molecular imprinting for drug bioanalysis. A review on the application of imprinted polymers to solid-phase extracton and binding assay. J. Chromatogr. B 2000, 739, 163-173.

20. Makote, R.; Collinson, M. M. Dopamine recognition in templated silicate films. Chem. Commun. 1998, 425-426.

21. Ling, T.-R.; Syu, Y. Z.; Tasi, Y.-C.; Chou, T.-C.; Liu, C.-C. Size-selective recognition of catecholoamines by molecular imprinting on silica-alumina gel. Biosens. Bioelectron. 2005, 21, 901-907.

22. Takeuchi, T.; Murase, N.; Maki, H.; Mukawa, T.; Shinmori, H. Dopamine selective molecularly imprinted polymers via post-imprinting modification. Org. Biomol. Chem. 2006, 4, 565-568.

23. Suedee, R.; Seechamnanturakit, V.; Canyuk, B.; Ovatlarnporn, C.; Martin, G. P. Temperature sensitive dopamine-imprinted ( $N, N$-methylene-bis-acrylamide cross-linked) polymer and its potential application to the selective extraction of adrenergic drugs from urine. J. Chromatogr. A 2006, 1114, 239-249.

24. Liu, K.; Wie, W. Z.; Zeng, J. X.; Liu, X. Y.; Gao, Y. P. Application of novel electrosynthesized polydopamine - imprinted film to the capacitive sensing of nicotine. Anal. Bioanal. Chem. 2006, 385, 724-729.

25. Liu, Y.; Wang, F.; Tan, T.; Lei, M. Study of the properties of molecularly imprinted polymers by computational and conformational analysis. Anal. Chim. Acta 2007, 581, 137-146.

26. Dineiro, Y.; Menendez, I.; Blanco-Lopez, M. C.; Lobo-Castanon, M. J.; Miranda-Ordieres, A. J.; Tunon-Blanco P. Computational predictions and experimental affinity distributions for a homovanillic acid amolecularly imprinted polymer. Biosens. Bioelectron. 2006, 22, 364-371.

27. Piletska, E. V.; Turner, N. W.; Turner, A. P. F.; Piletsky, S. A. Controlled release of the herbicide simazine from computationally designed molecularly imprinted polymers. J. Control. Release 2005, 108, 132-139.

28. Wie, S.; Jakusch, M.; Mizaikoff, B. Capturing molecules with templated materials - Analysis and rational design of molecularly imprinted polymers. Anal. Chim. Acta 2006, 578, 50-58.

29. Cormack, P. A. G.; Elorza, A. Z. Molecularly imprinted polymers: synthesis and characterisation. J. Chromatogr. B 2004, 804, 173-182.

30. Luliński, P.; Maciejewska, D.; Bamburowicz-Klimkowska, M.; Szutowski, M. Preliminary evaluation of molecularly imprinted polymer synthesized with dopamine hydrochloride as a template. Ninth Electronic Conference on Synthetic Organic Chemistry, ECSOC-9, Nov. 2005; B-001.

31. Ellwagner, A.; Berggren, C.; Bayoudh, S.; Crecenzi, C.; Karlsson, L.; Owens, P. K.; Ensing, K.; Cormack, P.; Sherrington, D.; Sellergren, B. Evaluation of methods aimed at complete removal of template from molecularly imprinted polymers. Analyst 2001, 126, 784-792.

32. Lakshmana, M. K.; Raju, T. R. An isocratic assay for norepinephrine, dopamine, and 5hydroxytryptamine using their native fluorescence by high-performance liquid chromatography with fluorescence detection in discrete brain areas of rat. Anal. Biochem. 1997, 246, 166-170. 
33. Sigma - Aldrich Co. Report. Eur. 2003, 9, 2.

34. HyperChem 7.02 program, HyperCube Inc, Waterloo, ON, Canada, 2002.

Sample Availability: Available from the authors.

(c) 2007 by MDPI (http://www.mdpi.org). Reproduction is permitted for noncommercial purposes. 